\title{
SYMMETRIZED SOLUTIONS FOR NONLINEAR STOCHASTIC DIFFERENTIAL EQUATIONS
}

\author{
G. ADOMIAN \\ Center for Applied Mathematics \\ University of Georgia \\ Athens, Georgia 30602 U.S.A. \\ and \\ L.H. SIBUL \\ Applied Research Laboratory \\ Pennsylvania State University \\ University Park, Pennsylvania 16802 U.S.A. \\ (Received February 15, 1980 and in revised form August 30,1980 )
}

ABSTRACT. Solutions of nonlinear stochastic differential equations in series form can be put into convenient symmetrized forms which are easily calculable. This paper investigates such forms for polynomial nonlinearities, i.e., equations of the form $L y+y^{m}=x$ where $x$ is a stochastic process and $L$ is a linear stochastic operator.

KEY WORDS AND PHRASES. Nonlinear stochastic differential equation, stochastic Green's function, polynomial nonlinearities and exponential nonlinearity.

\section{MATHEMATICS SUBJECT CLASSIFICATION CODES. 6OH}

\section{IINTRODUCTION.}

This paper extends some results for nonlinear stochastic differential equations in which first and second order statistical measures of the solution process were obtained in terms of stochastic Green's functions by a special iterative procedure [1]. The kernel of the integral which expresses the desired statistical measure of the dependent stochastic process in terms of the corresponding statistical measure of the forcing function and appropriate statistical measures of the 
stochastic coefficients of the differential equation is called the stochastic Green's function. This iterative method has been found to be effective in finding expressions for stochastic Green's functions because it does not require invalid closure approximations inherent in hierarchy methods and is not a perturbation method limited to small fluctuations. In this method higher order terms can be computed in terms of previously computed terms and the iteration can be stopped when the desired accuracy has been achieved. A new and convenient symmetrized form, (which is computationally very useful for polynomial nonlinearities) is derived for the solutions. The symmetric form means that any term of the series can be written immediately to assess its contribution.

By a stochastic differential equation, we mean one in which the differential operator is stochastic. (The forcing function and initial conditions are also allowed to be stochastic.) We are considering the general class of equations represented by $F y=x(t)$ where $F$ is a nonlinear stochastic operator. Assume $F$ is decomposable into a linear part $L$ and a nonlinear part $N$. Thus, we have $L y+N(y, \dot{y}, \ldots)=x(t)$ where $x(t)$ is a stochastic process on a suitable index space $T$ and probability space $(\Omega, F, \mu) ; L$ is a linear stochastic (differential) operator of nth order given by $L=\sum_{\nu=0}^{n} a_{v}(t) d^{\nu} / d t^{\nu}$ where one or more of the $a_{v}(t)$ for $\nu=0,1, \ldots, n-1$ may be stochastic processes on $T \times \Omega$, statistically independent of $x(t)$. In the earlier work [1], $N$ is a nonlinear term of the form $N=\sum_{\mu=0}^{m} b_{\mu}(t, \omega)\left(y^{(\mu)}\right)^{m \mu}$ where $y^{(\mu)}$ is the $\mu$ th derivative and $b_{\mu}$ may be stochastic processes for $\mu=0,1, \ldots, m$ on $T \times \Omega$. A physically reasonable assumption in many cases is that the processes $a_{\nu}, b_{\mu}$ are statistically independent of $x(t)$ for all $\mu, v$. It is further assumed that they are almost surely of class $C^{n}$ on $T$ for $\omega \in(\Omega, F, \mu)$ for allowed $\mu, \nu$. As in the earlier work, $L$ is decomposed into the sum $L+R$ where $R$ is a zero mean random operator and $L$ is deterministic, i.e., $L=\sum_{\nu=0}^{n}<a_{v}(t, \omega)>d^{\nu} / d t^{\nu}$ and $R=\sum_{\nu=0}^{n-1} \alpha_{v}(t, \omega) d^{v} / d t^{v}$ where $<a_{v}(t, \omega)>$ exists and is continuous on $T$. It is convenient for comparison with earlier work to assume $L y=x$ is a solvable (linear) equation; however, we could 
also begin with a solvable nonlinear equation as will be discussed elsewhere. We have now $L y=x-R y-N(y, \dot{y}, \ldots)$. Since we assume $L$ to be invertible, $y=L^{-1} x-L^{-1} R y-L^{-1} N(y, \dot{y}, \ldots)$; in terms of the Green's function $\ell(t, \tau)$ for $L, \quad y=\int_{0}^{t} \ell(t, \tau) x(\tau) d \tau-\int_{0}^{t} \ell(t, \tau) R[y(\tau)] d \tau-\int_{0}^{t} \ell(t, \tau) N(y(\tau), \dot{y}(\tau), \ldots) d \tau$.

The $L^{-1} R y$ term may involve derivatives in $R$; it is replaced with $\int_{0}^{t} R_{\tau}^{+}[\ell(t, \tau)] y(\tau, \omega) \mathrm{d} \tau$ where the adjoint operator $R^{+}$is given by

$$
R^{+}[\ell(t, \tau)]=\sum_{k=0}^{n-1}(-1)^{k}\left(d^{k} / d \tau^{k}\right)\left[\alpha_{k}(\tau) \ell(t, \tau)\right],
$$

assuming the stochastic bilinear concomitant (s.b.c.) vanishes. The latter is zero if the initial conditions are zero. In the case of random initial conditions, additional terms arise from the solution of the homogeneous equation and the value of the s.b.c. at $\tau=0$, where the s.b.c. is given by $[2,3]$.

$$
\begin{aligned}
& \left.\sigma(y(t, \omega) ; \ell(t, \tau))\right|_{\tau=0} ^{\tau=t} \\
& \quad=\left.\sum_{k=0}^{n-1} \sum_{\nu=0}^{k-1}(-1)^{\nu}\left[\ell(t, \tau) \alpha_{k}(\tau, \omega)\right]^{(\nu)}(k-1-\nu)(\tau, \omega)\right|_{\tau=0} ^{\tau=t}
\end{aligned}
$$

This expression vanishes at the upper limit because of a well known property of the Green's function $\ell(t, \tau)$ where $\ell(t, \tau)$ is the Green's function for $L$. For the linear case $(N=0)$ and if the s.b.c. vanishes, the equation for the solution process becomes

$$
y(t, \omega)=F(t, \omega)-\int_{0}^{t} R[l(t, \tau)] y(t, \omega) d \tau
$$

for which the solution has been given by the authors [1] in the Volterra integral form:

$$
\begin{aligned}
& y(t, \omega)=F(t, \omega)-\int_{0}^{t} \Gamma(t, \tau ; \omega) F(\tau, \omega) d \tau \\
& \Gamma(t, \tau ; \omega)=\sum_{m=1}^{\infty}(-1)^{m-1} K_{m}(t, \tau ; \omega) \\
& K_{m}(t, \tau ; \omega)=\int_{0}^{t} K\left(t, \tau_{1}\right) K_{m-1}(\tau,, \tau) d \tau 1 \\
& K_{1}=K=R[l(t, \tau)]
\end{aligned}
$$


The input process $x(t, \omega)$ is assumed to be bounded almost surely on $T$ and $\ell(t, \tau)$ is continuous on $T$; thus $|x(t, \omega)|<M_{1}$, a constant, or equivalently, $|F(t, \omega)|<M_{j}$, a constant. Further, the $\alpha_{v}$, for $v=0, \ldots, n-1$, are assumed to be bounded almost surely; in fact, the kth derivatives of the $\alpha_{v}$ are bounded for $k=0,1, \ldots, n-1$; i.e., $\left|\left(\partial^{k} / \partial t^{k}\right) \alpha_{\nu}(t)\right|<M_{2}$, a constant, for $t \varepsilon T, \omega \varepsilon(\Omega, F, \mu)$. This series is convergent under these conditions [4] (also discussed later in this paper). Mean square definitions can also be used. Derivatives in the differential equation and integrals in the Volterra integral equation must be in the same mean square, or almost sure, sense.

\section{SYMMETRIZED SOLUTIONS: QUADRATIC CASE}

We will assume $N(y, \dot{y}, \ldots)=N(y)$; i.e., no derivatives are involved. As a convenient special case for our first example, let $N(y)=b y^{2}$. We write $\sum_{i=0}^{\infty}(-1)^{i} \lambda^{i} y_{j}$ and $L=L+\lambda R+\lambda N$. The quantity $\lambda$ allows us to group terms more conveniently than in the previous work, yielding very convenient forms for computation. We have

$$
\begin{aligned}
y= & L^{-1} x-\lambda L^{-1} R y-\lambda L^{-1} b y^{2} \\
= & L^{-1} x-\lambda L^{-1} R \sum_{i=0}^{\infty}(-1)^{i} \lambda^{i} y_{i}-\lambda L^{-1} b \sum_{i=0}^{\infty} \sum_{j=0}^{\infty}(-1)^{i+j_{\lambda} \lambda^{i} \lambda^{j} y_{j} y_{j}} \\
= & y_{0}-\lambda L^{-1} R\left[y_{0}-\lambda y_{1}+\lambda^{2} y_{2} \ldots\right]-\lambda L^{-1} b\left[y_{0}-\lambda y_{1}+\lambda^{2} y_{2} \ldots\right] \\
& \cdot\left[y_{0}-\lambda y_{1}+\lambda^{2} y_{2} \ldots\right] \\
= & y_{0}-\lambda L^{-1} R\left[y_{0}-\lambda y_{1}+\lambda^{2} y_{2} \ldots\right]-\lambda L^{-1} b\left[y_{0}^{2}+\lambda^{2} y_{1}^{2}+\lambda^{4} y_{2}^{2} \ldots\right. \\
& \left.-2 \lambda y_{0} y_{1}+2 \lambda^{2} y_{0} y_{2}-2 \lambda^{3} y_{0} y_{3} \cdots-\lambda^{3} y_{1} y_{2}+\lambda^{4} y_{1} y_{3} \ldots\right] \\
= & y_{0}-\lambda L^{-1}\left[R y_{0}+b y_{0}^{2}\right]+\lambda^{2} L^{-1}\left[R y_{1}+2 b y_{0} y_{1}\right] \\
& -\lambda^{3} L^{-1}\left[R y_{2}+b y_{1}^{2}+2 b y_{0} y_{2}\right]
\end{aligned}
$$

Letting $\lambda=1$ and using initial conditions (see [2]), we obtain

$$
\begin{aligned}
& y_{0}=L^{-1} x \\
& y_{1}=L^{-1}\left[R y_{0}+b y_{0}^{2}\right]
\end{aligned}
$$




$$
\begin{aligned}
& y_{2}=L^{-1}\left[R y_{1}+b\left(y_{0} y_{1}+y_{1} y_{0}\right)\right] \\
& y_{3}=L^{-1}\left[R y_{2}+b\left(y_{1}^{2}+y_{0} y_{2}+y_{2} y_{0}\right)\right]
\end{aligned}
$$

These can be rewritten as

$$
\begin{aligned}
& y_{0}=L^{-1} x \\
& y_{1}=L^{-1}\left[R y_{0}+b y_{0} y_{0}\right] \\
& y_{2}=L^{-1}\left[R y_{1}+b\left(y_{0} y_{1}+y_{1} y_{0}\right)\right] \\
& y_{3}=L^{-1}\left[R y_{2}+b\left(y_{0} y_{2}+y_{1} y_{1}+y_{2} y_{0}\right)\right] \\
& \vdots \\
& y_{n+1}=L^{-1}\left[R y_{n}+b\left(y_{0} y_{n}+y_{1} y_{n-1}+y_{2} y_{n-2} \ldots y_{n} y_{0}\right)\right]
\end{aligned}
$$

or

$$
y_{n+1}=L^{-1} K_{n}\left(y_{0}\right)
$$

where $K_{n}\left(y_{0}\right)=R y_{n}+b\left(y_{0} y_{n}+y_{1} y_{n-1}+\ldots+y_{n} y_{0}\right)$ and $y=y_{0}-L^{-1} \Gamma\left(y_{0}\right)$ with $\Gamma\left(y_{0}\right)=\sum_{i=0}^{\infty}(-1)^{i} k_{j}\left(y_{0}\right)$. (The same letters $\Gamma$ and $k$ have been used as in the linear case but are identical only if $N=0$ and $\sigma=0$.)

That the convergence holds can be seen from the form of $k_{n}\left(y_{0}\right)$. In the 1 inear case convergence is obvious because when each $y_{i}$ is replaced by $y_{i-1}$ until $y_{0}$ is reached, and the quantities are replaced by their bounds as in our initial assumptions, we are left with the $n$-fold integral $\int d t$ and an $n !$ in the denominator as discussed in [4]. For the nonlinear case, if one examines the bracketed term $y_{0} y_{n}+\ldots y_{n} y_{0}$ and replaces each $y_{i}$ by $y_{i-1}$ until $y_{0}$ is reached, each term will yield a product of $n ! ' s$ in the demoninator. We now have $n$ such terms in the general term yielding $1 /(n-1) !$ and convergence follows. (The nonlinear part is analytic by assumption and leads to a finite number of terms in a Taylor expansion.)

\section{STATISTICAL MEASURES}

To obtain the mean or expected solution $\langle y\rangle$, the correlation $R_{y}\left(t_{1}, t_{2}\right)$, or the covariance $k_{y}\left(t_{1}, t_{2}\right)$, the solution process $y$ must, of course, be averaged over the appropriate probability space $\Omega$ to get $\langle y\rangle$. Similarly, 
$y\left(t_{1},\right)_{y}^{*}\left(t_{2}\right)$ is averaged to get $R_{y}\left(t_{1}, t_{2}\right)$. In the Zinear case (when $N=0$ ),

$$
\langle y\rangle=\langle F(t, \omega)\rangle-\int_{0}^{t}\langle\Gamma(t, \tau, \omega)\rangle\langle F(\tau)\rangle d \tau \text {. }
$$

In the nonlinear case (non-zero $N$ ), the stochastic coefficients $b_{\mu}(t, \omega)$ are assumed bounded almost surely on $T$ for $\omega \varepsilon(\Omega, F, \mu)$. The iteration now leads to extra terms arising from $L^{-1} N$, which are seen clearly in (1.1). As before, $y_{0}$ is bounded by hypothesis. For the nonlinear case, $y_{1}$ differs from the $y_{1}$ for the linear case by the addition of the term $L^{-1} b y_{0}^{2}$, i.e., by only terms involving $y_{0}$ in the $N(y)$ which we can denote by $N\left(y_{0}\right) . y_{2}$ differs from the $y_{2}$ for the linear case by addition of $L^{-1} b\left(y_{0} y_{1},+y_{1} y_{0}\right)$, i.e., by terms in $N(y)$ involving only $y_{0}, y_{1}$ which we can symbolize by $N\left(y_{0}, y_{1}\right)$, etc. for higher terms $y_{n}$.

$$
\begin{aligned}
y(t, \omega) & =F(t, \omega)-\int_{0}^{t} \Gamma(t, \tau ; \omega) F(\tau, \omega) d \tau-\int_{0}^{t} \ell(t, \tau) N(y, \dot{y}, \ldots) d \tau \\
& =F(t, \omega)-\int_{0}^{t} \Gamma(t, \tau ; \omega) F(\tau, \omega) d \tau-\int_{0}^{t} \ell(t, \tau) \sum_{\mu=0}^{m} b_{\mu}(\tau, \omega)(y(\mu))^{m_{\mu}} d \tau
\end{aligned}
$$

until $y_{0}$ is reached; ensemble averages again separate without closure approximations, etc., to determine first and second order statistics.

\section{CUBIC CASE}

Let us investigate whether symmetrized forms can be obtained for other than the quadratic case. We consider $N(y)=y^{3}$. Proceeding as before,

$$
\begin{aligned}
y= & L^{-1} x-L^{-1} R\left[y_{0}-\lambda y_{1}+\lambda^{2} y_{2}-\lambda^{3} y_{4}+\ldots\right]-\lambda L^{-1} b\left[y_{0}^{3}-\lambda\left(3 y_{0}^{2} y_{1}\right)\right. \\
& +\lambda^{2}\left(3 y_{0}^{2} y_{2}+3 y_{0} y_{1}^{2}\right)-\lambda^{3}\left(y_{1}^{3}+3 y_{0}^{2} y_{3}+6 y_{0} y_{1} y_{2}\right) \\
& \left.+\lambda^{4}\left(6 y_{0} y_{1} y_{3}+3 y_{0}^{2} y_{4}+3 y_{0} y_{2}^{2}+3 y_{1}^{2} y_{2}\right)\right] .
\end{aligned}
$$

In addition to the homogeneous solution, we get

$$
\begin{aligned}
& y_{0}=L^{-1} x \\
& y_{1}=L^{-1}\left[R y_{0}+b y_{0}^{3}\right] \\
& y_{2}=L^{-1}\left[R y_{1}+b\left(3 y_{0}^{2} y_{1}\right)\right]
\end{aligned}
$$




$$
\begin{aligned}
& y_{3}=L^{-1}\left[R y_{2}+b\left(3 y_{0}^{2} y_{2}+3 y_{0} y_{1}^{2}\right]\right. \\
& y_{4}=L^{-1}\left[R y_{3}+b\left(y_{1}^{3}+3 y_{0}^{2} y_{3}+6 y_{0} y_{1} y_{2}\right)\right] \\
& y_{5}=L^{-1}\left[R y_{4}+3 b\left(2 y_{0} y_{1} y_{3}+y_{0}^{2} y_{4}+y_{0} y_{2}^{2}+y_{1}^{2} y_{2}\right)\right]
\end{aligned}
$$

etc. It is difficult to see symmetry here immediately or derive convenient rules for writing the terms. We will return to these expressions shortly.

Returning to the quadratic case $N(y)=b y^{2}$, the solution can be given in an alternative form with the linear part separated out, writing $\Gamma_{\ell}$ and $\Gamma_{n}$ for the linear and nonlinear cases: then,

$$
y=F(t, \omega)-\int_{0}^{t} \Gamma_{\ell}(t, \tau ; \omega) F(\tau, \omega) d \tau-L^{-1} b \Gamma_{n}
$$

where $\Gamma_{n}=\sum_{n=0}^{\infty}(-1)^{n_{1}} k_{n}\left(y_{0}\right)$ and $k_{n}\left(y_{0}\right)$ is now given by $k_{n}\left(y_{0}\right)=y_{0} y_{n}+y_{1} y_{n-1}$ $+y_{n} y_{0}$. Thus,

$$
y=F(t, \omega)-\int_{0}^{t} \Gamma_{\ell}(t, \tau ; \omega) F(\tau, \omega) d \tau-\int_{0}^{t} \ell(t, \tau) b(\tau, \omega) \sum_{n=0}^{\infty}(-1)^{n} K_{n}\left(y_{0}\right) d \tau
$$

since each of the $y_{i}$ in $K_{n}\left(y_{0}\right)$ can be given in terms of $y_{i-1}$.

5. FOURTH POWER CASE

Let $N(y)=b y^{4}$. Then

$$
\begin{aligned}
y= & L^{-1} x-\lambda L^{-1} R\left[y_{0}-\lambda y_{1}+\lambda^{2} y_{2}-\lambda^{3} y_{3}-\lambda^{4} y_{4}+\ldots\right] \\
& -\lambda L^{-1} b\left[y_{0}^{4}-\lambda\left(4 y_{0}^{3} y_{1}\right)+\lambda^{2}\left(4 y_{0}^{3} y_{2}+6 y_{0}^{2} y_{1}\right)-\lambda^{3}\left(4 y_{0} y_{1}^{3}+3 y_{0}^{3} y_{1}\right.\right. \\
& \left.\left.+12 y_{0}^{2} y_{1} y_{2}+y_{0}^{3} y_{3}\right)+\lambda^{4}\left(12 y_{0}^{2} y_{1} y_{3}+12 y_{0} y_{1}^{2} y_{2}+6 y_{0}^{2} y_{2}^{2}+3 y_{0}^{3} y_{4}+y_{1}^{4}\right)\right] \\
y_{0}= & L^{-1} x \\
y_{1}= & L^{-1}\left[R y_{0}+b y_{0}^{4}\right] \\
y_{2}= & L^{-1}\left[R y_{1}+b\left(4 y_{0}^{3} y_{1}\right)\right] \\
y_{3}= & L^{-1}\left[R y_{2}+b\left(4 y_{0}^{3} y_{2}+6 y_{0}^{2} y_{1}^{2}\right)\right] \\
y_{4}= & L^{-1}\left[R y_{3}+b\left(4 y_{0} y_{1}^{3}+3 y_{0}^{3} y_{1}+12 y_{0}^{2} y_{7} y_{2}+y_{0}^{3} y_{3}\right)\right] \\
y_{5}= & L^{-1}\left[R y_{4}+b b_{4}\left(12 y_{0}^{2} y_{1} y_{3}+12 y_{0} y_{1}^{2} y_{2}+6 y_{0}^{2} y_{2}^{2}+3 y_{0}^{3} y_{4}+y_{1}^{4}\right)\right]
\end{aligned}
$$


The next section will clarify these last two cases.

6. POLYNOMIAL NONLINEARITIES

We now consider the case where $N(y)=b y^{m}$. Then

$$
y=L^{-1} x-\lambda L^{-1} R y-\lambda L^{-1} \text { by }{ }^{m} \text {. }
$$

Let $y=y_{0}+\lambda y_{1}+\lambda^{2} y_{2}+\ldots \lambda^{n} y_{n}+\ldots$ and assume $y^{m}=A_{0}+\lambda A_{1}+\lambda^{2} A_{2}+\ldots$ $+\lambda^{n} A_{n}+\ldots$ (We have previously assumed $y=\sum_{j=0}^{\infty}(-1)^{i} \lambda^{i} y_{i}$ but we get the same series in either case.) The $A_{0}, A_{1}, A_{2}, \ldots$ were found in a Danish paper in 1881 by Hansted [5]. The relations are:

$$
\begin{aligned}
A_{0} & =y_{0}^{m} \\
A_{1} & =m\left(y_{1} / y_{0}\right) A_{0}=m\left(y_{1} / y_{0}\right) y_{0}^{m}=m y_{0}^{m-1} y_{1} \\
2 A_{2} & =(m-1)\left(y_{1} / y_{0}\right) A_{1}+2 m\left(y_{2} / y_{0}\right) A_{0} \\
& =m(m-1) y_{0}^{m-2} y_{1}^{2}+2 m y_{2} y_{0}^{m-1} \\
3 A_{3} & =(m-2)\left(y_{1} / y_{0}\right) A_{2}+(2 m-1)\left(y_{2} / y_{0}\right) A_{1}+3 m\left(y_{3} y_{0}\right) A_{0} \\
\vdots & n A_{n}=(m-(n-1))\left(y_{1} / y_{0}\right) A_{n-1}+(2 m-(n-2))\left(y_{2} / y_{0}\right) A_{n-2}+(3 m-(n-3))\left(y_{3} / y_{0}\right) \\
& \cdot A_{n-3}+\ldots+n m\left(y_{n} / y_{0}\right) A_{0}
\end{aligned}
$$

Thus we have a systematic way of obtaining expansions for larger $m$. For smaller $m$ we can use the same method or simply multiply out the power series in $\lambda$ and collect terms of equal powers in $\lambda$. Both methods, of course, yield the same results. As an example, let $m=2$. We obtain

$$
\begin{aligned}
& A_{0}=y_{0}^{2}=y_{0} y_{0} \\
& A_{1}=m\left(y_{1} / y_{0}\right) y_{0}^{2}=2 y_{1} y_{0} \\
& A_{2}=y_{1} y_{1}+2 y_{2} y_{0} \\
& A_{3}=2 y_{1} y_{2}+2 y_{3} y_{0} \\
& A_{4}=2 y_{3} y_{1}+y_{2} y_{2}+2 y_{4} y_{0}
\end{aligned}
$$




$$
\begin{aligned}
& A_{5}=2 y_{5} y_{0}+2 y_{4} y_{1}+2 y_{2} y_{3} \\
& A_{6}=2 y_{0} y_{6}+2 y_{1} y_{5}+2 y_{2} y_{4}+y_{3} y_{3} \\
& \text { etc. }
\end{aligned}
$$

These can be put into symmetrized form where the indices of each term add to the index of $A_{n}$ and all possible sums are taken:

$$
\begin{aligned}
& A_{0}=y_{0} y_{0} \\
& A_{1}=y_{0} y_{1}+y_{1} y_{0} \\
& A_{2}=y_{0} y_{2}+y_{1} y_{1}+y_{2} y_{0} \\
& A_{3}=y_{0} y_{3}+y_{1} y_{2}+y_{2} y_{1}+y_{3} y_{0} \\
& A_{4}=y_{0} y_{4}+y_{1} y_{3}+y_{2} y_{2}+y_{3} y_{1}+y_{4} y_{0} \\
& A_{5}=y_{0} y_{5}+y_{1} y_{4}+y_{2} y_{3}+y_{3} y_{2}+y_{4} y_{1}+y_{5} y_{0} \\
& \vdots \\
& A_{n}=y_{0} y_{n}+y_{1} y_{n-1}+\ldots+y_{n-1} y_{1}+y_{n} y_{0}
\end{aligned}
$$

Our series obtained by equating equal powers of $\lambda$ is

$$
\begin{aligned}
& y_{0}=L^{-1} x \\
& y_{1}=-L^{-1}\left[R y_{0}+b A_{0}\right] \\
& y_{2}=-L^{-1}\left[R y_{1}+b A_{1}\right] \\
& \vdots \\
& y_{n+1}=-L^{-1}\left[R y_{n}+b A_{n}\right]
\end{aligned}
$$

where the $A_{n}$ are given above.

In the more general case of $y^{m}$,

$$
\begin{aligned}
& y_{0}=L^{-1} x \\
& y_{1}=-L^{-1}\left[R y_{0}+b A_{0}\right]=-L^{-1}\left[R y_{0}+b y_{0}^{m}\right]
\end{aligned}
$$




$$
\begin{aligned}
y_{2}= & -L^{-1}\left[R y_{1}+b A_{1}\right]=-L^{-1}\left[R y_{1}+b m y_{0}^{m-1} y_{1}\right] \\
\vdots & \\
y_{n+1}= & -L^{-1}\left[R y_{n}+b A_{n}\right] \\
= & -L^{-1}\left[R y_{n}+b\left\{(m-(n-1))\left(y_{1} / y_{0}\right) A_{n-1}+\left(2 m-(n-2)\left(y_{2} / y_{0}\right) A_{n-2}\right.\right.\right. \\
& \left.+\left(3 m-(n-3)\left(y_{3} / y_{0}\right) A_{n-3}+\ldots+n m\left(y_{n} / y_{0}\right) A_{0}\right\}\right]
\end{aligned}
$$

If $m=3$,

$$
\begin{aligned}
y_{0}= & L^{-1} x \\
y_{1}= & -L^{-1}\left[R y_{0}+b y_{0}^{3}\right] \\
y_{2}= & -L^{-1}\left[R y_{1}+b\left(3 y_{0}^{2} y_{1}\right)\right] \\
\vdots & \\
y_{n+1}= & -L^{-1}\left[R y_{n}+b\left\{3-(n-1)\left(y_{1} / y_{0}\right) A_{n-1}+(6-(n-2))\left(y_{2} / y_{0}\right) A_{n-2}\right.\right. \\
& \left.\left.+(9-(n-3))\left(y_{3} / y_{0}\right) A_{n-3}+\ldots+3 n\left(y_{n} / y_{0}\right) A_{0}\right\}\right]
\end{aligned}
$$

It is easily verified that these results for $m=3$ correspond to the earlier results given in 4; similarly, for $m=4$, we obtain the results of 5 .

We have noted that, if we write $y_{n+1}=L^{-1}\left[R y_{n}+b A_{n}\right]$, the expression for $A_{n}$ is simply obtained by writing all terms in which indices add up to $n$. But now the number of factors in each term is 3 , i.e., the same as $m$.

$$
\begin{aligned}
A_{0} & =y_{0} y_{0} y_{0} \\
A_{1} & =y_{0} y_{0} y_{1}+y_{0} y_{1} y_{0}+y_{1} y_{0} y_{0} \\
A_{2} & =y_{0} y_{0} y_{2}+y_{0} y_{2} y_{0}+y_{2} y_{0} y_{0}+y_{0} y_{1} y_{1}+y_{1} y_{0} y_{1}+y_{1} y_{1} y_{0} \\
A_{3} & =y_{1} y_{1} y_{1}+y_{0} y_{0} y_{3}+y_{0} y_{3} y_{0}+y_{3} y_{0} y_{0}+y_{0} y_{1} y_{2}+y_{1} y_{2} y_{0} \\
& +y_{2} y_{0} y_{1}+y_{1} y_{0} y_{2}+y_{0} y_{2} y_{1}+y_{2} y_{1} y_{0}
\end{aligned}
$$

etc.

Similarly for $m=4$, 


$$
\begin{aligned}
A_{0} & =y_{0} y_{0} y_{0} y_{0} \\
A_{1} & =y_{0} y_{0} y_{0} y_{1}+y_{0} y_{0} y_{1} y_{0}+y_{0} y_{1} y_{0} y_{0}+y_{1} y_{0} y_{0} y_{0} \\
A_{2} & =y_{0} y_{0} y_{0} y_{2}+y_{0} y_{0} y_{2} y_{0}+y_{0} y_{2} y_{0} y_{0}+y_{2} y_{0} y_{0} y_{0}+y_{0} y_{0} y_{1} y_{1} \\
& +y_{1} y_{1} y_{0} y_{0}+y_{0} y_{1} y_{0} y_{1}+y_{1} y_{0} y_{0} y_{1}+y_{0} y_{1} y_{1} y_{0}
\end{aligned}
$$

Thus, if $N(y)=b y^{m}$,

$$
y_{n+1}=L^{-1} R y_{n}+L^{-1} b A_{m, n}
$$

where $A_{m, n}$ is given by

$$
A_{m, n}=\sum_{i+j+k+\ldots+w} y_{i} y_{j} y_{k} \ldots y_{w}
$$

where $i+j+k+\ldots+w=n$ and the number or multiplicative factors is $m$. Hence, any polynomial nonlinearity is easily handled.

\section{EXAMPLE: APPROXIMATION FOR QUADRATIC NONLINEARITY}

As a simple example of the use of the preceding results for polynomial nonlinearities, we take the quadratic nonlinearity of 2 , i.e., the equation

$$
L y+y^{2}=x \text {. }
$$

We have $y=\sum_{i=0}^{\infty}(-1)^{i} y_{i}$ with

$$
y_{i+1}=L^{-1}\left[R y_{i}+A_{2, i}\right] \quad i \geq 0
$$

where $A_{m, n}$ has been defined in 6 . We now can compare various approximations of y.

First Stage Approximation

$$
y=y_{0}=L^{-1} x
$$

Second Stage Approximation

$$
y=y_{0}-L^{-1}\left[R y_{0}+A_{2,0}\right]=y_{0}-\left(L^{-1} R\right) y_{0}-L^{-1} y_{0}^{2}
$$

Third Stage Approximation 


$$
y=\sum_{i=1}^{3}(-1)^{i} y_{i}=y_{0}-y_{1}+y_{2}
$$

where

$$
\begin{aligned}
& y_{1}=L^{-1} R y_{0}+L^{-1} A_{2,0}=L^{-1} R y_{0}+L^{-1} y_{0}^{2} \text { and } \\
& y_{2}=L^{-1} R y_{1}+L^{-1} A_{2,1}=L^{-1} R y_{1}+L^{-1}\left(y_{0} y_{1}+y_{1} y_{0}\right) .
\end{aligned}
$$

Thus,

$$
\begin{aligned}
y= & y_{0}-\left(L^{-1} R y_{0}+L^{-1} y_{0}^{2}\right)+\left(L^{-1} R\right)\left[\left(L^{-1} R\right) y_{0}+L^{-1} y_{0}^{2}\right]+L^{-1} y_{0}\left[\left(L^{-1} R\right) y_{0}\right. \\
& \left.+L^{-1} y_{0}^{2}\right]+L^{-1}\left[\left(L^{-1} R\right) y_{0}+L^{-1} y_{0}^{2}\right] y_{0}
\end{aligned}
$$

Finally,

$$
\begin{aligned}
y= & y_{0}-\left(L^{-1} R\right) y_{0}-L^{-1} y_{0}^{2}+\left(L^{-1} R\right)\left(L^{-1} R\right) y_{0}+2\left(L^{-1} R\right) L^{-1} y_{0}^{2} \\
& +\left(L^{-1} R\right) L^{-1} y_{0}^{2}+2 L^{-1} L^{-1} y_{0}^{3} .
\end{aligned}
$$

Fourth Stage Approximation

$$
y=\sum_{i=0}^{3}(-1)^{i} y_{i}=y_{0}-y_{1}+y_{2}-y_{3}
$$

We need to compute only $y_{3}$ since the previous terms have been calculated.

$$
\begin{aligned}
y_{3}= & L^{-1} R y_{2}+L^{-1} A_{2,2} \\
= & L^{-1} R y_{2}+L^{-1}\left(y_{0} y_{2}+y_{1} y_{1}+y_{2} y_{0}\right) \\
= & L^{-1} R\left[L^{-1} R y_{1}+L^{-1} A_{2,1}\right]+L^{-1} y_{0}\left[L^{-1} R y_{1}+L^{-1} A_{2,1}\right] \\
& +L^{-1} y_{1} y_{1}+L^{-1}\left[L^{-1} R y_{1}+L^{-1} A_{2,1}\right] y_{0} \\
= & \left(L^{-1} R\right)\left(L^{-1} R\right)\left(L^{-1} R y_{0}+L^{-1} y_{0}^{2}\right]+L^{-1} R L^{-1} A_{2,1}+L^{-1} y_{0} L^{-1} R\left[L^{-1} R y_{0}\right. \\
& \left.+L^{-1} y_{0}^{2}\right]+L^{-1} y_{0} L^{-1} A_{2,1}+L^{-1}\left[L^{-1} R y_{0}+L^{-1} y_{0}^{2}\right]\left[L^{-1} R y_{0}+L^{-1} y_{0}^{2}\right] \\
& +L^{-1} L^{-1} R\left[L^{-1} R y_{0}+L^{-1} y_{0}^{2}\right] y_{0}+L^{-1} L^{-1} A_{2,1} y_{0} \\
y_{3}= & \left(L^{-1} R\right)\left(L^{-1} R\right)\left(L^{-1} R\right) y_{0}+\left(L^{-1} R\right)\left(L^{-1} R\right) L^{-1} y_{0}^{2}+\left(L^{-1} R\right) L^{-1}\left(y_{0} y_{1}+y_{1} y_{0}\right) \\
& +L^{-1}\left(L^{-1} R\right)\left(L^{-1} R\right) y_{0}^{2}+L^{-1}\left(L^{-1} R\right) L^{-1} y_{0}^{3}+L^{-1} y_{0} L^{-1}\left(y_{0} y_{1}+y_{1} y_{0}\right) \\
& \left.+L^{-1}\left(L^{-1} R\right)\left(L^{-1} R\right) y_{0}^{2}+L^{-1}\left(L^{-1} R\right) y_{0}^{3}+L^{-1}\left(L^{-1} R\right) y_{0}^{3}+L^{-1} L^{-1} y_{0}^{4}\right]
\end{aligned}
$$




$$
\begin{aligned}
& +L^{-1}\left(L^{-1} R\right)\left(L^{-1} R\right) y_{0}^{2}+L^{-1}\left(L^{-1} R\right) L^{-1} y_{0}^{3}+L^{-1} L^{-1}\left(y_{0} y_{1}+y_{1} y_{0}\right) y_{0} \\
= & \left(L^{-1} R\right)\left(L^{-1} R\right)\left(L^{-1} R\right) y_{0}+\left(L^{-1} R\right)\left(L^{-1} R\right) L^{-1} y_{0}^{2}+\left(L^{-1} R\right) L^{-1} y_{0}\left[\left(L^{-1} R\right) y_{0}\right. \\
& \left.+L^{-1} y_{0}^{2}\right]+\left(L^{-1} R\right) L^{-1}\left[\left(L^{-1} R\right) y_{0}+L^{-1} y_{0}^{2}\right] y_{0}+L^{-1}\left(L^{-1} R\right)\left(L^{-1} R\right) y_{0}^{2} \\
& +L^{-1}\left(L^{-1} R\right) L^{-1} y_{0}^{3}+L^{-1} L^{-1} y_{0}^{2}\left[\left(L^{-1} R\right) y_{0}+L^{-1} y_{0}^{2}\right]+L^{-1} L^{-1}\left[\left(L^{-1} R\right) y_{0}\right. \\
& \left.+L^{-1} y_{0}^{2}\right] y_{0}^{2}+L^{-1}\left(L^{-1} R\right)\left(L^{-1} R\right) y_{0}^{2}+L^{-1} L^{-1}\left(L^{-1} R\right) y_{0}^{3}+L^{-1} L^{-1} L^{-1} y_{0}^{4} \\
& +L^{-1}\left(L^{-1} R\right)\left(L^{-1} R\right) y_{0}^{2}+L^{-1}\left(L^{-1} R\right) L^{-1} y_{0}^{3}+L^{-1} L^{-1} y_{0}\left[\left(L^{-1} R\right) y_{0}+L^{-1} y_{0}^{2}\right] y_{0} \\
& +L^{-1} L^{-1}\left[\left(L^{-1} R\right) y_{0}+L^{-1} y_{0}^{2}\right] y_{0}^{2} .
\end{aligned}
$$

We obtain

$$
y_{3}=\left(L^{-1} R\right)^{3} y_{0}+6\left(L^{-1} R\right)^{2} L^{-1} y_{0}^{2}+9\left(L^{-1} R\right) L^{-1} L^{-1} y_{0}^{3}+5 L^{-1} L^{-1} L^{-1} y_{0}^{4} .
$$

\section{EXPONENTIAL NONLINEARITY}

Consider $N(y)=e^{y}$ :

$$
L y+e^{y}=x
$$

By the symmetric method of solution, we must resort to a Taylor expansion for $e^{y}$, i.e., $y+y^{2} / 2 !+y^{3} / 3 !+\ldots$; hence, the computation becomes tedious if sufficient terms of the Taylor expansion are to be used for a reasonable approximation of $e^{y}$. Let us take four terms for the Taylor expansion; $e^{y \approx y} y y^{2} / 2 !+y^{3} / 3$ ! $+y^{4} / 4 !$. Then, by the symmetric method,

$$
y=\sum_{i-1}^{\infty}(-1)^{i} y_{i-1} \text { for } i \geq 1
$$

with

$$
y_{i}=L^{-1} R y_{i-1}+L^{-1} A_{m, i-1}
$$

where $m$ is the power of the polynomial term. We realize we would have to compute quite a large number of terms. For example, we saw how many terms we had to compute at the fourth stage of approximations for $y^{2}$; we would have to do the same thing for $y$ and $y^{3}$ as well. The inverse method or operator theoretic method which appear elsewhere $[5,6]$ doesn't need such decompositions for expressions like $e^{y}$ or $\log y$, so is actually more convenient for such cases; however, 
the symmetric method is convenient for the polynomial cases.

ACKNOWLEDGEMENT: This work is supported by a Sloan Foundation grant for which the authors express their appreciation.

\section{REFERENCES}

1. ADOMIAN, G. "Nonl inear Stochastic Differential Equations." J. Math. Anal. \& Appl., vol. 55, no. 2, August 1976, 441-452.

2. ADOMIAN, G., and LYNCH, T. E. "Stochastic Differential Operator Equations with Random Initial Conditions." J. Math. Anal. \& App Z., vol. 61, no. 1, November 1, 1977, 216-226.

3. MILLER, K. S. Theory of Differential Equations. Norton Co., New York (1959).

4. ADOMIAN, G. "Random Operator Equations in Mathematical Physics, Part I." Joumal of Math. Physics, vol. 11, no. 3, (March, 1970), 1069-1084.

5. HANSTED, B. "Nogle Bemaerkniger om Bestemmelsen af Koefficienterne I m'Te Potens af en Potensraekke." Pidsskrift for Mathematik, vol. 4, no. 5, $1881,12-16$.

6. ADOMIAN, G., and MALAKIAN, K. "Operator-Theoretic Solution of Nonl inear Stochastic Systems." J. Math. Anal. \& App Z., vol. 76, no. 1, July 1980, 183-201. 


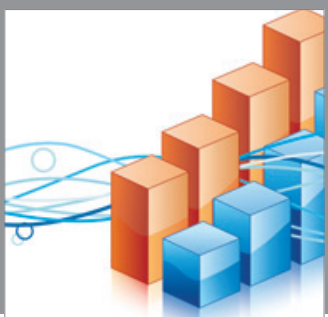

Advances in

Operations Research

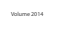

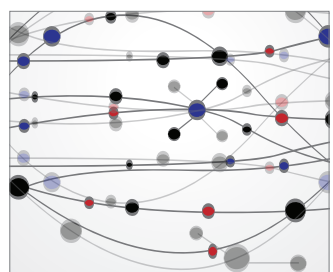

\section{The Scientific} World Journal
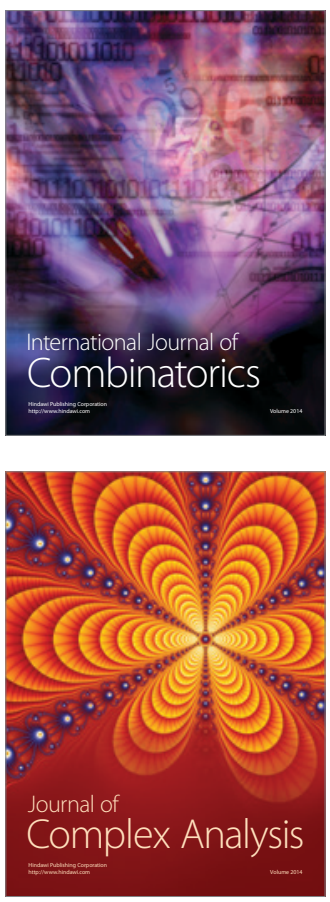

International Journal of

Mathematics and

Mathematical

Sciences
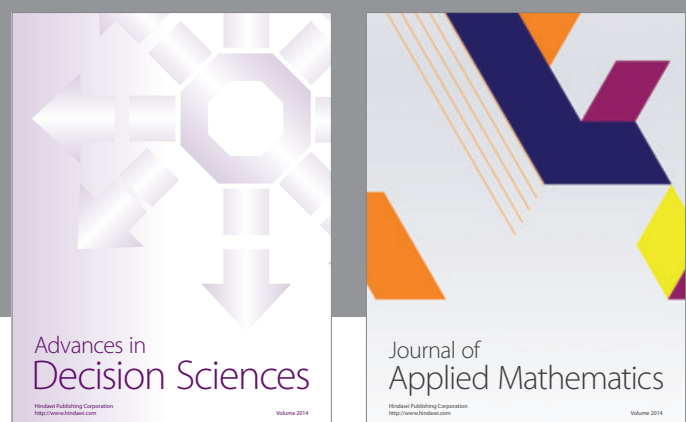

Journal of

Applied Mathematics
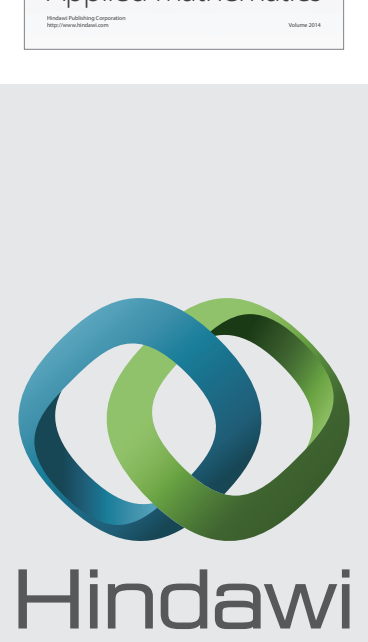

Submit your manuscripts at http://www.hindawi.com
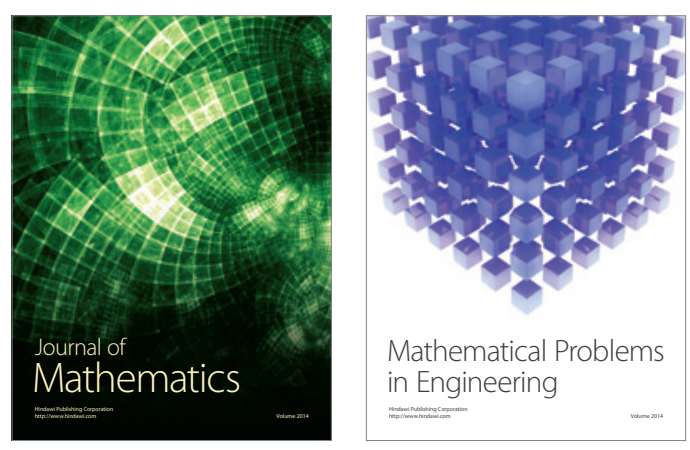

Mathematical Problems in Engineering
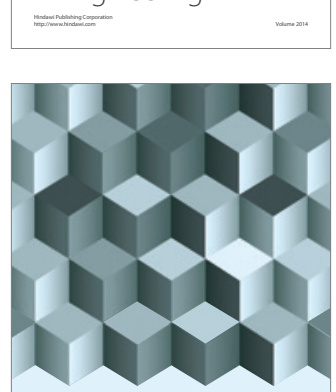

Journal of

Function Spaces
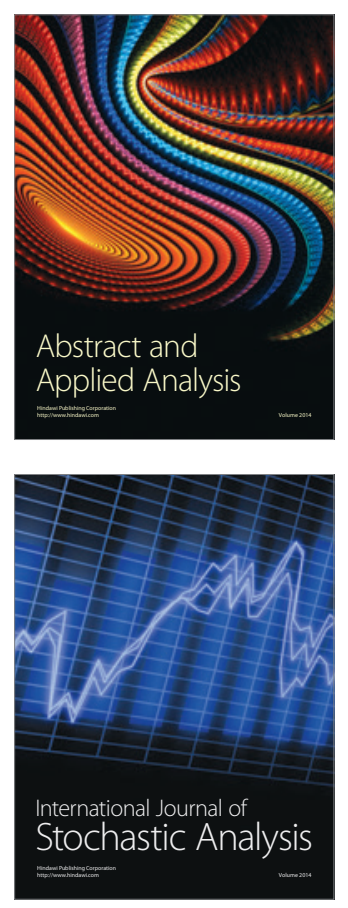

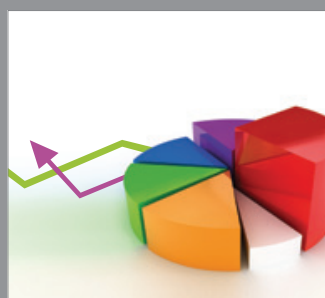

ournal of

Probability and Statistics

Promensencen
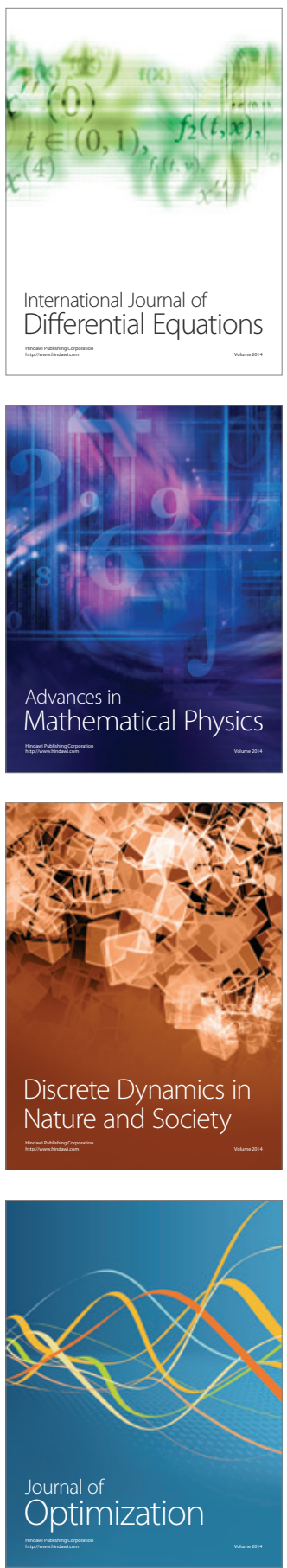